\title{
Free Convection and Irreversibility Analysis inside a Circular Porous Enclosure
}

\author{
Shohel Mahmud ${ }^{1}$ and Roydon Andrew Fraser ${ }^{2}$ \\ ${ }^{1}$ Department of Mechanical Engineering, University of Waterloo, Waterloo, Ontario \\ Canada. Tel. +1 519888 4567; Fax +1 519888 6197; Email: smahmud@uwaterloo.ca \\ ${ }^{2}$ Email: rafraser@uwaterloo.ca
}

Received: 5 November 2002 / Accepted: 11 May 2003 / Published: 31 December 2003

\begin{abstract}
We investigate the nature of heat transfer and entropy generation for natural convection in a two-dimensional circular section enclosure. The enclosure is assumed to fill with porous media. The Darcy momentum equation is used to model the porous media. The full governing differential equations are simplified with the Boussinesq approximation and solved by a finite volume method. Whereas the Prandtl number Pr is fixed to 1.0. Results are presented in terms of Nusselt number, entropy generation number, and Bejan number.
\end{abstract}

Keywords: porous cavity, irreversibility, entropy generation, Bejan number, Nusselt number

\section{Introduction}

Horizontal cylinders filled with fluids are commonly encountered in the world around us. This type of geometry and flow configuration are commonly observed in the field of electronics, cooling system, heat exchanger, etc. When their side wall temperature is non-uniform, natural convection motion develops inside the cylinder and many efforts have been devoted, over the last decades, to understand the flow structure and related heat transfer mechanism under various heating conditions. Articles by Sierra [1], Ostrach and Hantman [2], Ostrach [3], Xin et al. [4] are some of them. Two configurations have been extensively studied: the configuration heated from below (Rayleigh-Benard convection) and that heated from the side. Most of the previous work deals with non-porous media and none of them considered a Second-law (of thermodynamics) analysis. 
Therefore, in the present work, we study the entropy generation characteristics along with the nature of heat transfer inside a porous circular cavity by solving numerically the fully nonlinear momentum and energy equations in a two-dimensional Cartesian frame. More specifically, the cavity is divided into two symmetrical parts by the vertical centerline and both of the parts are perfectly isothermal, but differentially heated. Results are presented for different Rayleigh numbers ( $R a=10$ to 5000).

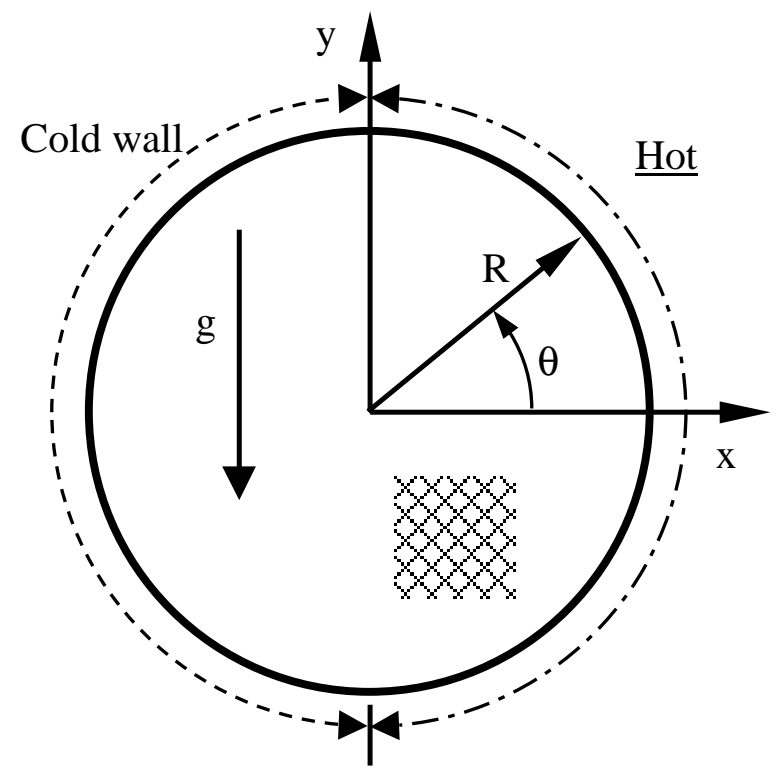

Figure 1. Schematic diagram of the problem under consideration

\section{Equations and Numerical Methods}

Figure 1 shows the domain to be analyzed and the adopted coordinate system. All asterisked quantities in this paper are in dimensional form. The left symmetrical part of the cylinder is cold and the right part is hot as indicated in Figure 1. It is assumed that the cavity is completely filled with the fluid. Uneven density of fluid originating from the temperature difference of the walls produces buoyancy. The saturated porous medium is assumed to be isotropic in thermal conductivity and follows the Darcy model (see Bejan [5]). Finally, the set of non-dimensional governing equations in terms of the stream function $\psi$ and temperature $\Theta$ are

$$
\begin{gathered}
\frac{\partial^{2} \psi}{\partial x^{2}}+\frac{\partial^{2} \psi}{\partial y^{2}}=R a \frac{\partial \Theta}{\partial x}, \\
\frac{\partial \Theta}{\partial \tau}+\frac{\partial \psi}{\partial y} \frac{\partial \Theta}{\partial x}-\frac{\partial \psi}{\partial x} \frac{\partial \Theta}{\partial y}=\frac{\partial^{2} \Theta}{\partial x^{2}}+\frac{\partial^{2} \Theta}{\partial y^{2}}
\end{gathered}
$$




$$
\begin{aligned}
& x=\frac{x^{*}}{D}, y=\frac{y^{*}}{D}, \psi=\frac{\psi^{*}}{\alpha}, \Theta=\frac{T-T_{0}}{\Delta T}, \Delta T=T_{\mathrm{hot}}-T_{\mathrm{cold}}, T_{0}=\frac{T_{\mathrm{hot}}+T_{\mathrm{cold}}}{2} \\
& \tau=t\left(\frac{\alpha}{W^{2} \sigma}\right), R a=\frac{g K \beta \Delta T D}{\alpha \nu}, u=\frac{\partial \psi}{\partial y}, \nu=-\frac{\partial \psi}{\partial x}, \sigma=\frac{\phi \rho C_{p}+(1-\phi) \rho_{s} C_{s}}{\rho C_{p}},
\end{aligned}
$$

subjected to the following boundary conditions

$$
\begin{aligned}
& R=0.5,-\frac{\pi}{2} \leq \theta<\frac{\pi}{2}: \psi=0 \text { and } \Theta=0.5 \\
& R=0.5, \frac{\pi}{2} \leq \theta<-\frac{\pi}{2}: \psi=0 \text { and } \Theta=-0.5 .
\end{aligned}
$$

Equations (1) and (2) along with the boundary conditions given in Eq. (4) are solved using control volume based Finite-Volume method. A non-staggered and non-uniform grid system is used with a higher mesh density near the walls. TDMA solver solves discretized and linearized equation systems. The whole computational domain is subdivided by an unequally spaced mesh with a size of $116 \times 128$.

\section{Entropy Generation}

For the porous media, which follows the Darcy model, the dimensionless form of the local rate of entropy generation $\left(N_{S}\right)$ can be calculated from the following equation:

$$
N_{S}=\frac{S_{g e n}^{\prime \prime \prime}}{S_{0}^{\prime \prime \prime}}=\left[\left(\frac{\partial \Theta}{\partial x}\right)^{2}+\left(\frac{\partial \Theta}{\partial y}\right)^{2}\right]+\frac{E c_{m} \times \operatorname{Pr}}{\Omega}\left[\left(\frac{\partial \psi}{\partial x}\right)^{2}+\left(\frac{\partial \psi}{\partial y}\right)^{2}\right],
$$

where $S_{g e n}^{\prime \prime \prime}$ and $S_{0}^{\prime \prime \prime}$ are local entropy generation rate and the characteristics transfer rate (see Bejan [6]), respectively. The detailed derivation of the above equation is available in Bejan [5]. Equation (5) consists of two parts. The first part (first square bracketed term at the right-hand side of Eq. (5)) is the irreversibility due to finite temperature gradient and generally termed as heat transfer irreversibility (HTI). The second part is the contribution of fluid friction irreversibility (FFI) to entropy generation, which can be calculated from the second square bracketed term. Bejan number $(B e)$ can be mathematically expressed as

$$
B e=\frac{\mathrm{HTI}}{\mathrm{HTI}+\mathrm{FFI}}
$$

\section{Results and Discussion}

We first present the flow and thermal fields' behavior in terms of streamlines and isothermal lines. Isothermal lines inside the cavity are shown in Figure 2 for six different values of Rayleigh number as indicated in the figure. Corresponding streamfunction plots are shown in Figure 3. Conduction like isotherms are observed at $R a=10$. Streamlines are similar to concentric circles except near the center region of the cavity, where elliptic core is observed. With the increase of Rayleigh number, convection current develops inside the cavity and isothermal lines start to swirl as shown in Figures 2(a) and 2(b). 
Core of the streamlines rotate counterclockwise direction with the increasing $R a$. Thermal spots appear near the bottom half of the hot wall and the top half of the cold wall at $R a=500$. Temperature gradient,

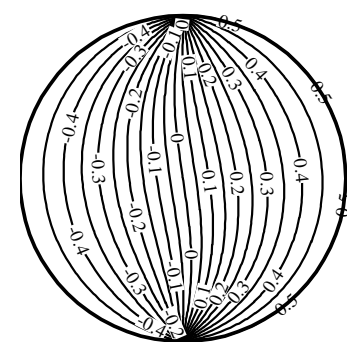

(a) $\mathrm{Ra}=10$

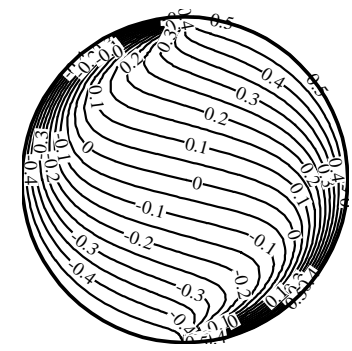

(d) $\mathrm{Ra}=500$

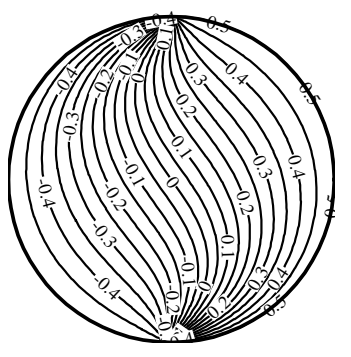

(b) $\mathrm{Ra}=50$

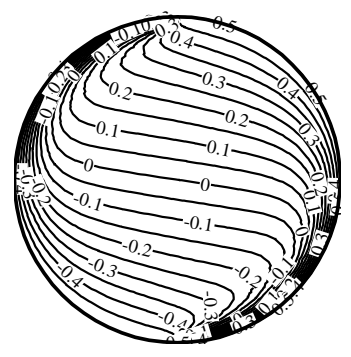

(e) $\mathrm{Ra}=1000$

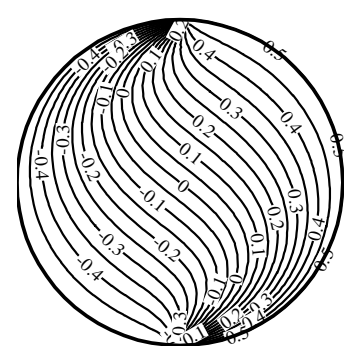

(c) $\mathrm{Ra}=100$

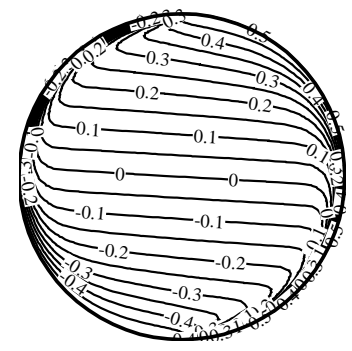

(f) $\mathrm{Ra}=5000$

Figure 2. Isothermal lines at different Rayleigh number

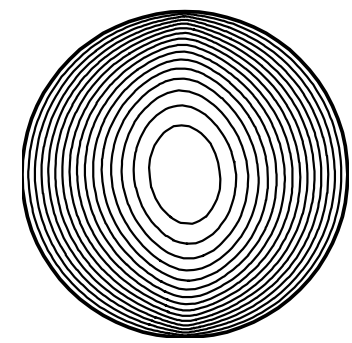

(a) $\mathrm{Ra}=10$

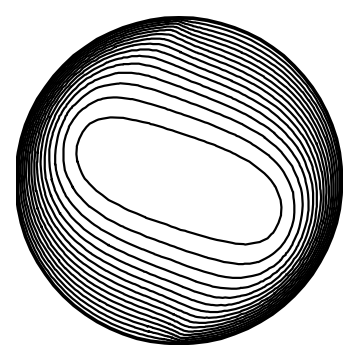

(d) $\mathrm{Ra}=500$

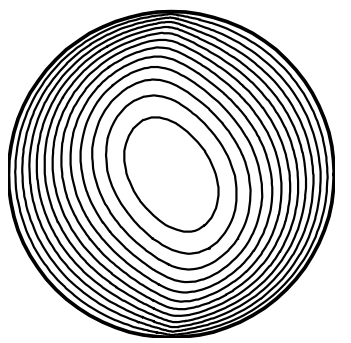

(b) $\mathrm{Ra}=50$

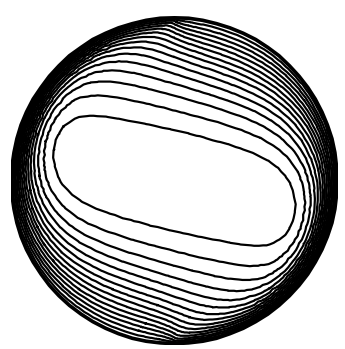

(e) $\mathrm{Ra}=1000$

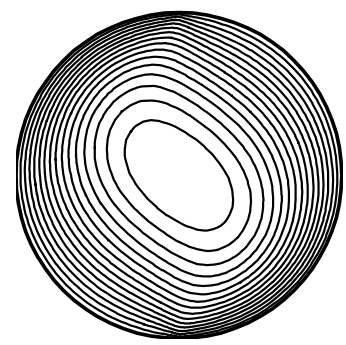

(c) $\mathrm{Ra}=100$

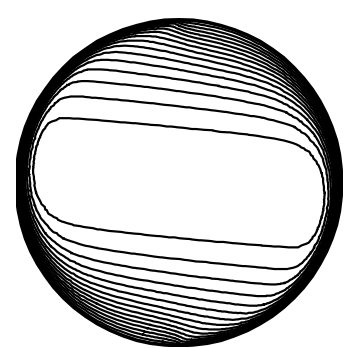

(f) $\mathrm{Ra}=5000$

Figure 3. Streamlines at different Rayleigh number 
as well as, heat transfer rate is higher in magnitude near these thermal spots. Core of the streamlines elongated at this Rayleigh number. Further increase in the Rayleigh number elongates the thermal spots along the wall. Boundary layer type of flow is observed at $R a=5000$. Fluid is almost stagnant at the

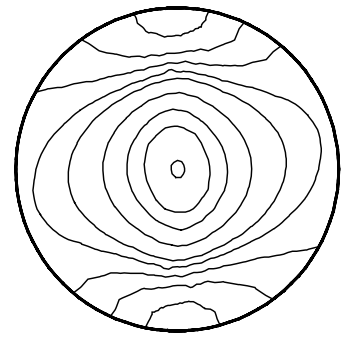

(a) $\mathrm{Ra}=10$

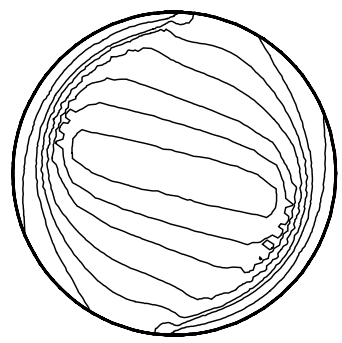

(d) $\mathrm{Ra}=500$

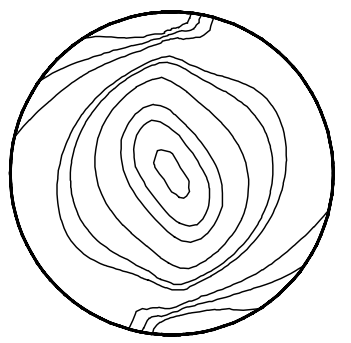

(b) $\mathrm{Ra}=50$

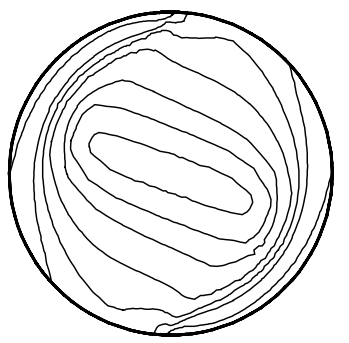

(e) $\mathrm{Ra}=1000$

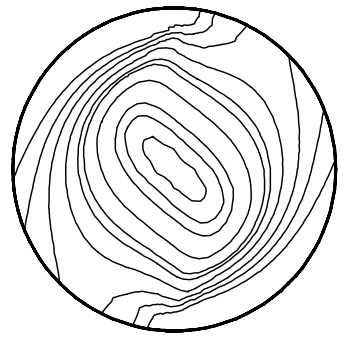

(c) $\mathrm{Ra}=100$

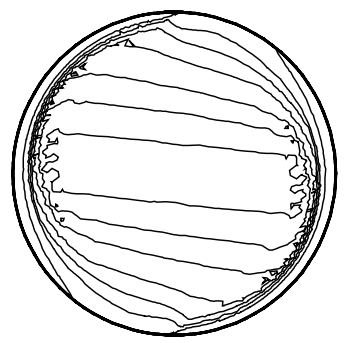

(f) $\mathrm{Ra}=5000$

Figure 4. Isentropic lines at different Rayleigh number

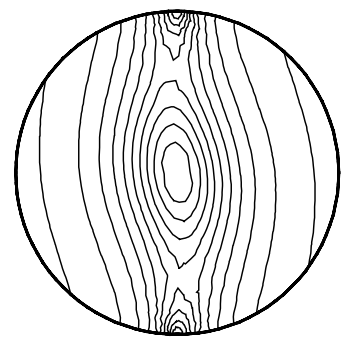

(a) $\mathrm{Ra}=10$

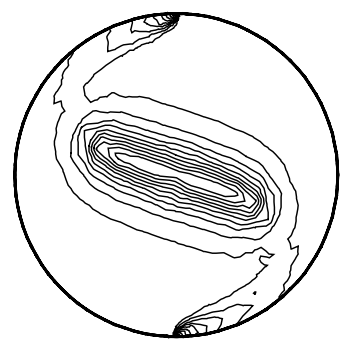

(d) $\mathrm{Ra}=500$

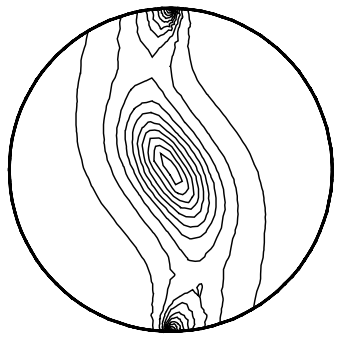

(b) $\mathrm{Ra}=50$

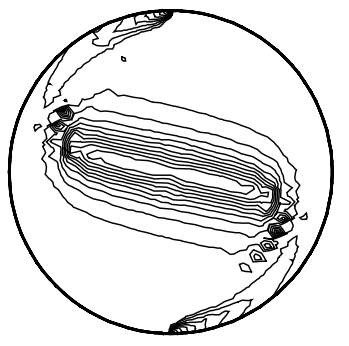

(e) $\mathrm{Ra}=1000$

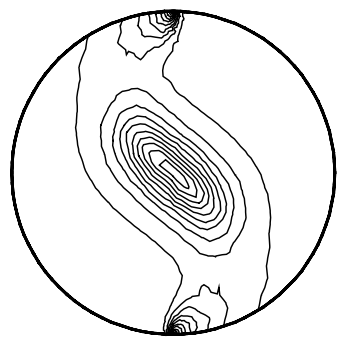

(c) $\mathrm{Ra}=100$

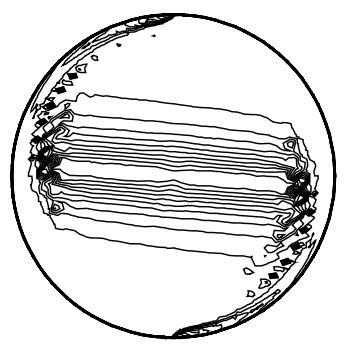

(f) $\mathrm{Ra}=5000$

Figure 5. Iso-Bejan lines at different Rayleigh number 
middle portion of the cavity. For the same flow configuration contours of entropy generation number and Bejan number are plotted in Figures 4(a)-(f) and Figures 5(a)-(f). At low Rayleigh number, entropy generation rate mainly dominated by the finite temperature gradient. The extent of irreversibility throughout the whole cavity is observed. With increasing Rayleigh number, convection current dominates and the extent of irreversibility start to concentrate towards the wall. At high Rayleigh number, high concentration of entropy generation is observed near the wall. However, heat transfer irreversibility (Bejan number) shows a different picture. With increasing Rayleigh number, higher concentration of Bejan number is observed at the center region of the cavity and the extent of the heat transfer irreversibility rotates along the direction of convective distortion of isothermal lines.

Average Nusselt number and entropy generation number at steady state are calculated using the following equation:

$$
\begin{aligned}
& N u_{\text {av,steady }}=\left.\frac{1}{\pi R} \int_{S} \frac{\partial \Theta}{\partial n}\right|_{\text {wall }} d s, \\
& N s_{a v, \text { steady }}=\frac{1}{\forall} \int_{\forall} N s d s,
\end{aligned}
$$

where $s$ is the distance along the circular wall, $S$ is equal to $\pi R$, and $\forall$ is representing the volume of the cavity. Figure 6 shows the distribution of average Nusselt number and entropy generation number as a function of Rayleigh number.

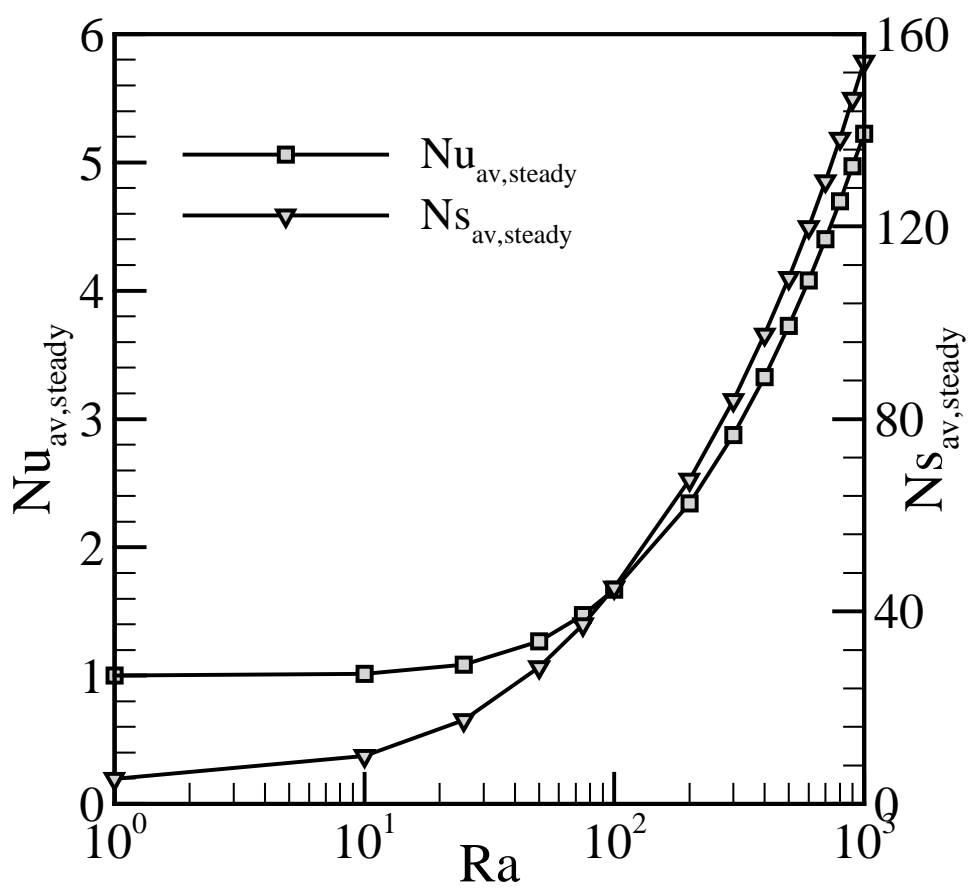

Figure 6. Average Nusselt and entropy generation numbers as a function of Rayleigh number 


\section{Nomenclature}

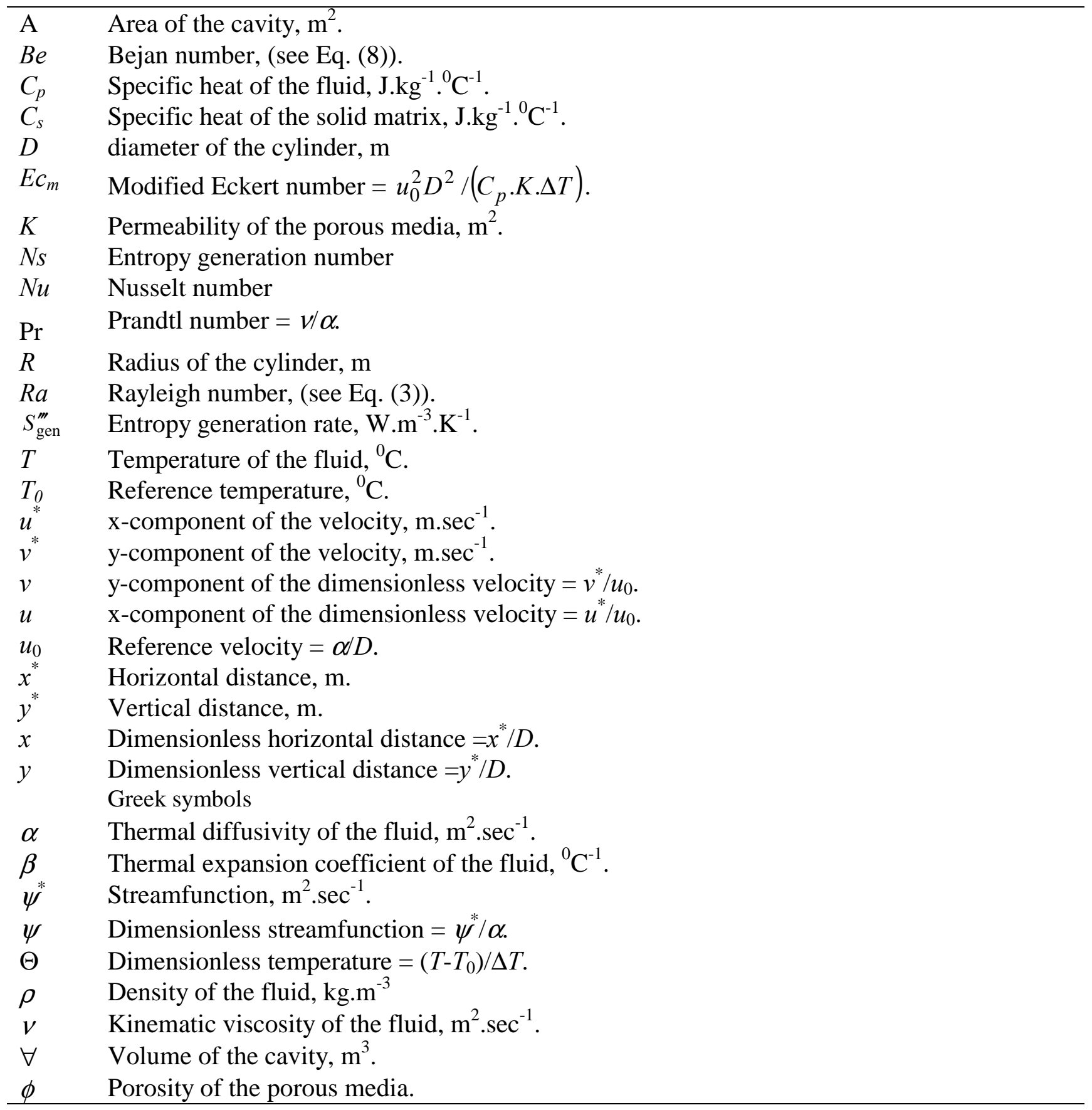

\section{Conclusions}

The nature of heat transfer, entropy generation, and heat transfer irreversibility inside a differentially heated circular cylinder is presented in this paper. In conduction regime, both average Nusselt number and the entropy generation number are independent of Rayleigh number variation. In convection 
dominated regime, these parameters show an increasing tendency with increasing Rayleigh number. At high Rayleigh number, the near-wall magnitude of overall entropy generation rate is higher, but heat transfer irreversibility is higher at the center portion of the cavity.

\section{Reference}

[1] Sierra, F.Z. Steady natural convection in a cylindrical cavity. Int. Comm. Heat Mass Transfer. 2002, 29, 213-221.

[2] Ostrach, S., Hantman, R.G. Natural convection inside a horizontal cylinder. Chem. Engr. Commun. 1981, 9, 213-243.

[3] Ostrach, S. Natural convection in enclosure. J. Heat Transfer, 1988, 110, 1175-1190.

[4] Xin, S., Quere, P.L., Daube, O. Natural convection in a differentially heated horizontal cylinder: Effects of Prandtl number on flow structure and instability. Physics of Fluids. 1997, 9, 1014-1032.

[5] Bejan, A. Convection Heat Transfer (John Wiley \& Sons, New York, 1984).

[6] Bejan, A. Entropy Generation Minimization (CRC Press, New York, 1996).

(C) 2003 by MDPI (http://www.mdpi.org). Reproduction for noncommercial purposes permitted. 